\title{
ÜBER BILDUNG UND ZERFALL VON WÄNDEN
}

\author{
Eduard Gerber
}

Fels gilt als Inbegriff der Beständigkeit. Wenn etwas felsenfest ist, dann wankt es nicht, und wer auf Fels gebaut, hat wohl gebaut. Auf schwer zugänglichen Felsen wurden im Mittelalter Burgen gebaut, und wenn sie auch zerfallen sind, so hat sich doch der Fels kaum verändert. Mancher markante Felsgipfel wird von einer Kirche oder Kapelle gekrönt, und durch Jahrhunderte kann man zu ihnen aufschauen. Die römische Kirche ist auf Fels gebaut.

Diese Bilder und Begriffe der Festigkeit und Unveränderlichkeit zeigen, daß sich im menschlichen Leben, ja darüber hinaus durch Jahrhunderte, wenn nicht Jahrtausende, die Felsformen kaum sichtbar wandeln. Selbst Felsen, die kühn überhängen oder wie morsche Ruinen aussehen, können Jahrhunderte überdauern, ehe sie in Trümmer zerfallen. Steinschläge sind zwar eine häufige, unter Umständen in besonderem Gestein eine nahezu alltägliche Erscheinung, aber Felsstürze oder gar größere Bergstürze gehören doch zu den seltenen Ereignissen.

Wenn wir nun auch sicher wissen, daß Felswände einmal entstanden sind und vergehen werden, so sind die damit verknüpften Vorgänge der direkten Beobachtung weitgehend entzogen. Fels hat für unsere Beobachtung eine feste, scharf umrissene Form. Auf die Erfassung der Form ist deshalb auch die Feldbeobachtung weitgehend beschränkt.

Die Bildung und der Zerfall sind vorwiegend physikalische, mechanische Vorgänge. Experimentell lassen sie sich nur ungenügend studieren, weil die langen Zeiträume, über die die Natur verfügt, nicht übertragbar sind. Wände werden aber nicht nur von Fels gebildet, sondern auch von wesentlich weniger festen Materialien, in welchen die Vorgänge der Bildung und des Zerfalls so schnell ablaufen, daß direkte Beobachtung möglich wird. Es ist deshalb sinnvoll, auch solche Wände in die Untersuchung einzubeziehen.

Die moderne Entwicklung der Ingenieurbauten hat dazu geführt, daß auch von dieser Seite der Problemkreis in Angriff genommen wurde. Eine Felsmechanik ist in Entwicklung begriffen. Die Beschäftigung mit dem Fels zeigt, daß es sich beim Zerfall nicht einfach nur um eine reine Verwitterungserscheinung handelt, durch welche Kruste um Kruste zermürbt wird und abstürzt, sondern daß im Fels an bezeichnenden Stellen Spannungen vorhanden sind, welche zu Zerreißungen führen. Ja es ist sogar anzunehmen, daß der Fels durch sehr lange Zeiträume Verformungen erfährt und unter Umständen Fließvorgänge beteiligt sind.

In diesem Aufsatz, welcher frühere Arbeiten über alpine Morphologie (1956/1959) fortsetzt und ein Teilproblem einer größeren Untersuchung über Form und Bildung von Gehängen in einem ersten Bericht darzustellen versucht, wird Wert darauf gelegt, auf die Vielgestaltigkeit der Formen und der möglichen Vorgänge hinzuweisen. Als vorläufige Mitteilung aus dem Rahmen einer größeren Untersuchung und in der Beschränkung auf den verfügbaren Platz in dieser Zeitschrift trägt der Aufsatz den Charakter einer Skizze. Eine ausführliche Behandlung des ganzen Problemkreises ist einer späteren Arbeit vorbehalten.

\section{Wände als Gehänge}

Wände sind eine besondere Art von Gehänge. Im strengen Sinn des Wortes müßten es senkrechte Gehänge sein. Doch werden auch sehr steile Gehänge, die aber $90^{\circ}$ nicht erreichen, dazugerechnet. Es ist also ein Grenzwert zu suchen, der diese Gehängeform von andern scheidet.

Zum Begriff «Wand» gehört die Entblößung. In der Wand tritt das Innere des Berges nackt zu Tage. Das Gegenteil ist verdeckt. Bei dieser Decke kann es sich nur um lockern Schutt, um Verwitterungsmaterial handeln, der normalerweise die Oberflächenformen bildet und den Fels verhüllt und im humiden Klima und nicht zu großer Höhe meist Vegetation trägt. Aus den beiden Eigenschaften «steil» und «nackt» ergibt sich ein erster Ansatz für die Definition: Wände sind Gehänge, auf denen Lockerschutt nicht liegenbleibt. Diese Feststellung ist etwas zu präzisieren. Denn jedem Kletterer ist bekannt, daß viele Felswände Lockerschutt enthalten, der, einmal in Bewegung gesetzt, den gefährlichen Steinschlag ergibt. So können wir erweitern: Wände sind Gehänge, auf denen einmal in Bewegung gesetzter Lockerschutt nicht zur Ruhe kommt, sondern frei abstürzt oder abgleitet. Gegensatz hiezu sind Gehängeflächen, auf denen Schutt nicht nur liegenbleibt, sondern sich auch anhäufen kann. Die steilsten Gehänge, auf denen Lockerschutt liegenbleibt, sind offensichtlich die Schutthalden. 
Sie entstehen am Fuß von Wänden. Schutt, der aus diesen stürzt und gleitet, häuft sich darauf an. Schutthalden haben einen maximalen Böschungswinkel, der nicht überschritten werden kann. Er ist abhängig von der Form und Rauhigkeit des Materials. Zahlreiche Messungen, die schon an trockenen Schutthalden durchgeführt wurden (z.B. Piwowar 1903, Stiny 1925, Freise 1932), ergaben für den maximalen Böschungswinkel Werte zwischen $27^{\circ}$ und $43^{\circ}$, die kleinsten in Tonschiefern, die größten in rauhbrüchigem Granit. Sobald jedoch Schutthalden verdichtet sind, was erstaunlich oft der Fall ist, sind auch in Schutthalden kleine Wände möglich, die allerdings meist rasch zerfallen.

Halten wir als erstes fest: Wände sind schuttfreie Oberflächen, deren Neigung größer sein muß als der Maximalwinkel frischer Schutthalden. Dieser Maximalwinkel ist in der Reihe verschieden geneigter Gehängeflächen ein wichtiger Grenzwinkel, der Flächen mit ganz verschiedener Entwicklung trennt, denn in und auf Gehängen mit Schuttdecke verlaufen die Verwitterung und der Materialtransport ganz anders als auf nackten Oberflächen.

Überschreitet die Gehängeneigung je nach Material $30-40^{\circ}$, so kann sich auf der Oberfläche der Verwitterungsschutt nicht anhäufen, die Oberfläche bleibt nackt. Dies ist eine erste brauchbare Feststellung, die allerdings die Erscheinung noch nicht klärt, daß die meisten Wände wesentlich steiler sind, eben, wie wir feststellten, $W$ ände bilden.

Ganz allgemein sollen im folgenden Gehänge, die Schutt tragen, Flachhänge und solche, welche steiler sind als der Schutthaldenwinkel, Steilhänge genannt werden.

\section{Der maximale Böschungswinkel und die maximale $W$ andhöhe}

Wird ein kohäsionsloses Material wie Gehängeschutt, Kies oder Sand frei aufgeschüttet, so bildet sich eine natürliche Böschung, die einen durch das Material bestimmten maximalen Winkel nicht überschreitet. In einem Hang, in welchem z. B. Kalke mit Kalkmergeln, Mergeln und Tonen wechsellagern, bilden die Kalke die steilsten, die Mergel und Tone die flachern Böschungen. Unverkennbar bilden besonders resistente Gesteine die höchsten und schroffsten Felsen. Die Idee liegt deshalb nahe, für jedes Gestein einen maximalen Böschungswinkel zu postulieren. Das Problem hat die Morphologen mehrfach beschäftigt, und die These hat Befürwortung, aber auch Ablehnung gefunden.

Zur Abklärung ist die bekannte Gruppierung in Lockergesteine und Festgesteine heranzuziehen. Ferner ist zu unterscheiden zwischen kohäsionslosem Lockergestein und kohärentem Lockergestein.

Der maximale Böschungswinkel von kohäsionslosem Lockergestein, wie trockenem Sand, ist eine unbestrittene, eindeutig feststellbare und meßbare Größe.

Die meisten Lockergesteine sind nicht kohäsionslos. Feuchter Sand kann durchaus kleine Wändchen bilden. In jeder Kiesgrube kann man Wände beobachten. Lößwände beträchtlicher Höhe und guter Standfestigkeit sind eine verbreitete Erscheinung, und in Lehmgruben sind Lehmwände möglich. All diese Materialien können nicht nur Vertikalwände, sondern sogar kleine Überhänge bilden. Materialabhängig ist nicht die Oberflächenneigung des kohärenten Materials, wohl aber die Höhe einer steilen Böschung oder Wand.

Wird in Wänden in kohärentem Lockergestein eine materialabhängige Höhe überschritten, so geht die Wand zu Bruch. Bei diesem Vorgang entstehen, wie später auszuführen sein wird, meist wieder neue vertikale Wandstücke, allerdings von geringerer Höhe. Das abgestürzte und abgeglittene Material bildet am neuen Wandfuß Haldenformen, die je nach Sturz- und Gleithöhe, aber auch nach Material und Durchnässungszustand ganz verschieden aussehen und verschieden geneigt sein können.

Daß auch in der Bodenmechanik immer noch von einem maximalen Böschungswinkel kohärenter Gesteine gesprochen wird, trotzdem dieser Begriff den Tatsachen widerspricht, geht aus einer Bemerkung von Terzaghi (1954) hervor: «In manchen Lehrbü- 
chern sind sogar 'Tabellen mit Angaben über den natürlichen Böschungswinkel bindiger Böden enthalten, obwohl der natürliche Böschungswinkel von der Böschungshöhe abhängig ist.»

Bei kohärentem Gestein ist bis zu einer materialabhängigen Höhe jede Böschungsneigung, Wände und Überhänge inbegriffen, standfest.

Die Rückbildung und der Zerfall von Steilhängen und Wänden im bindigen Lockergestein ist ein Verwitterungsvorgang. Daß dieser im Lockergestein verhältnismäßig rasch vor sich geht, hat mit dem Problem des maximalen Böschungswinkels an sich nichts zu tun. Mit der Verwitterung werden wir uns noch eingehend zu befassen haben.

\section{Die Lage der $W$ and im Gehänge}

Um eine Übersicht über die Vielgestaltigkeit der Wandformen zu gewinnen, empfiehlt sich eine Einteilung nach der Lage im Gehänge und nach dem Verhältnis zum Gegengehänge. Eine Wand entwickelt sich nicht gleich und hat meist nicht das gleiche Aussehen, ob sie das ganze Gehänge einnimmt, von ausgedehntem Flachgehänge überhöht ist oder solchem aufruht. Vor allem aber sind meist große Formunterschiede vorhanden zwischen Wänden, die einen Grat bilden, sich also mit einer Gegenwand verschneiden, und solchen, welche oben durch einen Flachhang oder eine Plateaufläche abgeschlossen sind, also zwischen gratbildenden und gratlosen Wänden.

Im allgemeinen sind die Vorgänge in den gratbildenden Wänden komplizierter. Es wird denn auch in dieser Arbeit ein Hauptgewicht auf die Entwicklung gratloser Wände gelegt. Die Vielgestalt gratbildender Wände kann nur gestreift werden.

\section{Gratlose Wände}

Wird die Wand oben durch einen Flachhang oder eine Plateaufläche abgeschlossen, so kann über dem Wandscheitel der Verwitterungsschutt liegenbleiben und eine normale Verwitterung bis zur Bodenbildung stattfinden. Von oben stürzt also Verwitterungsschutt nur im Maß der Rückwitterung der Wand ab. Die Wände können das ganze Gehänge bilden und vom Gehänge$\mathrm{fu} ß$ bis zur Wasserscheide hinaufreichen. Eine unten durch eine Horizontalebene, oben durch eine Plateaufläche begrenzte Wand hat Lehmann und Bakker als Modell für ihre Theorie der Rückwitterung gedient. Durch das Rückwittern bildet sich am Hangfuß eine Halde, wenn der Wandschutt nicht durch ein Gewässer fortlaufend weggeschwemmt wird.

Die Wand mit ihrer Schutthalde kann aber auch einem ausgedehnten Flachhangsockel aufruhen oder wiederum von einem ausgedehnten Flachhang überhöht sein. Dann ist die Entwicklung der Wand mit der Entwicklung des Flachhanges verknüpft. Fließrorgänge im Flachhang können die Wand in Mitleidenschaft ziehen, und fluviale Zerschneidung kann auf die Wand übergreifen. Ebenso kann die Entwicklung eines selbständigen Entwässerungsnetzes im Flachhang über der Wand in diese übergreifen und die Wand von oben her anschneiden und gliedern.

Endlich kann die Wand auch als Band zwischen Flachhängen angeordnet sein. Wände im Gehänge können horizontal verlaufen oder im Gehänge talabwärts auf- oder absteigen. Solche Felsbänder können durch die Gehängezerschneidung gegliedert sein, sie können bastionsartig vortreten. Der Zerfall kann auch schon so stark fortgeschritten sein, daß nicht mehr von Felswänden, sondern nur noch von felsdurchsetztem Hang gesprochen werden kann.

Felsbänder mit Flachhangpartien können mehrmals wechseln, wie z. B. im Hang des nördlichen Walenseeufers. Den Übergang zu gratbildenden Felswänden bilden Wände, die sich mit einem flachen Gegenhang verschneiden. Beim Rückwittern nimmt bei dieser Anordnung die Höhe ab.

\section{Gratbildende Wände}

Gratbildende Wände unterscheiden sich grundsätzlich von gratlosen dadurch, daß die beiden sich verschneidenden Wände in ihrer Entwicklung miteinander verkoppelt sind. Bei der Verwitterung im Bereich des Grates kann loses Material nicht liegenbleiben, sondern stürzt in die Wand. Dadurch wird diese erniedrigt und natürlich stets beidseitig, auf welche Seite auch immer das Material stürzt. Ein Grateinschnitt wirkt sich meist auf beiden Seiten als Steinschlagrinne aus.

Gratformen bilden Wasserscheiden. Selten beginnen sie am Gehängefuß, meist sind sie mehr oder weniger ausgedehnten Flachhängen aufgesetzt. Als Wasserscheiden zwischen zwei Tälern steigen sie meist talaufwärts an und kulminieren in Gratgipfeln.

Sie können aber auch innerhalb eines ausgedehnten Gehänges auftreten und sind dann vom rückwärtigen Gehänge durch eine Mulde abgetrennt oder laufen senkrecht zur Talachse über das Gehänge hinunter. Als Übergangsformen von gratlosen zu gratbildenden Wänden sind Pfeiler 
und Zacken aufzufassen, die beim Rückwittern einer gratlosen Wand sich aus dieser gelöst haben und vor ihr stehengeblieben sind.

\section{V orgänge in Wänden}

Bei der Bildung und der Rückbildung der Wände spielen sich bestimmte singuläre Vorgänge $a b$, die mit der Wand als Form zusammenhängen und in andern Gehängearten nicht in dieser charakteristischen Art beobachtet werden können. Dies gilt schon für die Vorgänge, die unter dem Begriff «Verwitterung» im engern Sinn verstanden werden. Denn in Wänden muß ja die Verwitterung definitionsgemäß unvollständig verlaufen, da das sich bildende Lockermaterial abstürzt und abgeschwemmt wird. Immer wieder wird frisches Gestein freigelegt und neu der Verwitterung ausgesetzt. Eine Verwitterung bis zur Bodenbildung findet nicht statt.

$\mathrm{Zu}$ dieser Verwitterung im engern Sinn kommen nun noch Vorgänge, die mit der Wand in ganz besonderer Weise verknüpft sind und mit den Spannungsverhältnissen, der Dynamik steiler und hoher Flächen zusammenhängen.

\section{Wände in kohärentem Lockermaterial}

Wände in kohärentem Lockermaterial gehören nicht zu den Formen, die zur Charakterisierung des alpinen Hochgebirges besonders aufgezählt werden, denn sie bilden keine jener wild aufstrebenden und eindrucksvollen Felsformen. Und doch spielen sie überall dort, wo ein Gewässer sich rasch in Lockermaterial einschneidet, eine nicht unbedeutende Rolle. Sehr eindrücklich ist eine Fahrt mit der Rhätischen Bahn durch die Schlucht des Rheins, welche er in den viele Kubikkilometer umfassenden Bergsturz von Flims eingeschnitten hat. Auch Moränenmaterial, Gehängeschutt und fluviale Schotter können so stark verfestigt sein, daß sie, vor allem im Talgrund, hohe Wände bilden.

Das Studium solcher Wände bietet den Vorteil, daß die Bildung und der Zerfall in kurzen Zeiträumen beobachtet werden kann. Noch schneller wickeln sich die Vorgänge innerhalb künstlicher Gruben in wenig verfestigten Kiesmassen ab. Hier zeigen oft auch kleine Rinnsale, die sich bei starken Niederschlägen bilden und über ein Kiesbord fließen, wie rasch schluchtartig angeordnete Kieswände sich bilden können. Beim Abbau in tiefen Kiesgruben wird künstlich nachgemacht, was der Fluß am Fuß einer Kieswand vollbringt: die Wand wird am Fuß unterschnitten, bis es zu Nachstürzen kommt. Je nach dem Verfestigungsgrad sind kleine Überhänge möglich, besonders in gut verdichteten sandhaltigen oder gar tonhaltigen Partien. Abrisse solcher Überhänge erfolgen entlang sehr steiler bis vertikaler Flächen. Die Wand stürzt nur dann nicht steil nach, wenn eine schiefverlaufende Gleitfläche schon vorher vorhanden ist.

Wächst die Wand, so werden bei einer bestimmten Höhe am Fuß der W and Werte erreicht, welche die Druckfestigkeit des Kieses übersteigen. Dadurch geht der Fuß der Wand zu Bruch, der darauf lagernde Kies verliert seine Stütze, die Wand bricht sehr steil nach. Am Fuß häuft sich der Kies als unverfestigter, kohäsionsloser Schutt mit einer Neigung von rund $30^{\circ}$ an. Wird die Schutthalde nicht wegtransportiert, so schützt sie den darunter befindlichen Fuß vor weiterem Zerfall.

$\mathrm{Zu}$ diesen großen Abbrüchen kommt die Wandverwitterung und Rückwitterung im kleinen. Die Entblößung immer wieder frischer Wandteile erlaubt keine tiefgreifende chemische Verwitterung. Da die Kohäsion vom Wassergehalt abhängt, zerfällt sie, wenn das Porenwasser verdunstet. An heißen Tagen prasseln von Kieswänden fast ununterbrochen Steine nieder und wirbelt Staub hoch, wenn die äußerste Schicht austrocknet. Bei starkem Regenwetter kann umgekehrt aus der Wand Wasser austreten und zu Erosionserscheinungen Anlaß geben.

Durch den Absturz ganzer Wandschichten und durch die Austrocknungsverwitterung wächst die Schutthalde am Wandfuß zunehmend in die Höhe. Die Wandrückwitterung ist abgeschlossen, wenn die Schutthalde den Wandscheitel erreicht hat. Bis dahin gibt es zwei gegensätzliche Gehängeformen, die nahezu senkrechte Wand und die 
Schutthalde von etwa $30^{\circ}$, die kaum durch $Z$ wischenglieder miteinander verbunden sind.

Was hier von Kieswänden, oft in Wechsellagerung mit Sandbändern, geschildert wurde, gilt offenbar auch für reine Sandwände. Aus der «Monographie d'une paroi de sablière», von Y. Guillien (1960) sei hier als charakteristische Beobachtung festgehalten: «La paroi recule parallèlement à elle-même.» Der Autor unterscheidet «la dégradation corticale du tail»- der typische Verwitterungsvorgang in Wänden - und die Fließvorgänge und Abspülungen im Schuttfuß.

Wand und Schuttfuß sind korrelate Formen, in denen sich ganz verschiedene Vorgänge abspielen, sowohl bei der Verwitterung wie auch beim Abtrag.

Im allgemeinen wird Sand und Kies nicht zu den kohärenten Lockergesteinen gerechnet. Im trockenen Zustand sind diese Materialien sogar Typen kohäsionsloser Lokkergesteine.

Schon die feinern Psamite, der Mehlsand mit o,2-0,02 mm Korngröße, behalten beim Austrocknen eine gewisse Restfeuchtigkeit und erhalten eine große Festigkeit. Hierher gehört z. B. der Löß, der allerdings auch noch feinere Fraktionen enthält. Beim Schluff mit o,o2-0,0002 mm macht sich der Einfluß der sorbierten Wasserhüllen noch stärker bemerkbar, besonders aber bei der Schwebfraktion von weniger als $0,0002 \mathrm{~mm}$.

Dabei ist nicht notwendig, daß das Material gleichkörnig ist. Moränen mit hohem Anteil an pelitischem Material können Fraktionen bis zu Blockgröße aufweisen und trotzdem eine große Festigkeit haben, wie z. B. die Erdpyramiden zeigen.

Beim Austrocknen zerfällt bindiges Lockergestein nicht mehr in Einzelkörner. Je nach dem Material entstehen regelmäßige Trockenrisse und kleine Schuppen oder Plättchen. Bei der Wasseraufnahme quillt das Material, bei Frost blättern geschichtete Tone auf.

Pelite können in verschiedenen Zustandsformen vorliegen, wobei die Unterschiede durch den verschiedenen Wassergehalt bedingt sind. 1. Sie können so gut geschichtet und durch die überlagernden Schichten so großen Drucken ausgesetzt gewesen sein, daß sie sich anisotrop verhalten und durch die große Vorverdichtung der Wassergehalt ein Minimum darstellt. Man kann dann von Schiefertonen sprechen. So ist z. B. der Opalinuston des Juragebirges vorzüglich geschichtet und enthält gut eingeregelte Glimmerplättchen. Zur Schichtung tritt hier ein senkrecht dazu verlaufendes Kluftsystem.

2. Dem Material kann eine ausgesprochene Schichtung fehlen (wie bei gewissen Gehängelehmen), der Wassergehalt kann aber trotzdem so gering sein, daß sie eine große Festigkeit aufweisen und verhältnismäßig hohe Wände möglich sind.

3. Das Material kann so viel Wasser enthalten, daß es sich gut plastisch verhält und leicht knetbar ist.

4. Endlich kann es breiartig fließfähig sein.

Hier sollen nur die zwei Zustandsformen besprochen werden, die Wände bilden können.

Die kritische Höhe einer Wand läßt sich nach der Formel $h_{c}=4 \frac{c}{\gamma}$ berechnen, worin c die Kohäsion in $t / \mathrm{m}^{2}$ und $\gamma$ das Raumgewicht in $\mathrm{t} / \mathrm{m}^{3}$ bedeutet. Setzen wir bei Ton als Wert für c 3,5 ein, für $\gamma 1,75$, so erhalten wir als kritische Höhe ungefähr $8 \mathrm{~m}$.

Es soll zunächst das Verhalten einer Wand innerhalb dieser Höchstgrenze betrachtet werden.

In einer Baugrube von 3,5 m Tiefe im Gehängelehm mußten infolge der großen Kälte des Winters 1962/63 die Arbeiten für längere Zeit eingestellt werden. Da konnte beobachtet werden, wie sich, vom Fuß der Wand her beginnend, Platten von einigen Zentimetern Dicke und einiger $\mathrm{dm}^{2}$ Fläche lösten. In mehreren Schichten schritt der Vorgang von unten nach oben fort. Die obern Abrißränder verliefen bogenförmig-gewölbeartig. Im obersten Viertel blieb ein bis zu $20 \mathrm{~cm}$ vorragender Überhang stehen.

Die Plattenbildung kann in diesem Fall durch Frostsprengung erklärt werden. Für poröses, feuchtes Material beschreibt Föppl (1958) Versuche, aus denen er schließt, daß nicht das sich bildende Eis allein sprengend wirkt, sondern daß durch die Eisbildung das im Gesteinsinnern eingeschlossene Porenwasser unter Spannung gerät. Das im Gestein vorhandene Wasser sucht sich beim Gefrieren einen Ausweg in Richtung der größten 
Nachgiebigkeit des Gesteinsmassivs durch die in dieser Richtung verlaufenden Gesteinskanäle und beansprucht dabei das Gestein in Flächen senkrecht zu Strömungsrichtung auf $Z$ ug, so daß sich die Schichten parallel zur freien Oberfläche ausbilden ...»

Daß der Vorgang am Fuß beginnt und die Platten von unten nach oben fortgesprengt werden, hat mit der Frostverwitterung und Plattenbildung an sich nichts zu tun. Der Vorgang ist nur aus den I)ruckverhältnissen in der Wand verständlich, welche am Wandfuß am größten sind. Werden die Platten von unten her losgelöst, so hängen sie frei von oben und reißen auf $Z$ ug. Beim Abreißen entlasten sie die Überlagerung des obersten Wandteiles, die dadurch stabil wird.

Zu einem völlig andern Verlauf kommt es, wenn eine Wand im kohärenten Material die kritische Höhe erreicht. Der obere Teil der Wand steht dabei unter Zugspannung. Es bilden sich Zugrisse, die senkrecht verlaufen und normalerweise bis etwa zur halben Höhe der Wand herabreichen ('Terzaghi 1954). Durch diese Zugrisse wird das Material geschwächt, in verstärktem Maß, wenn durch Ansammlung von Oberflächenwasser in den Zugrissen die Gleichgewichtsbedingungen verändert werden. Dadurch wird die Scherfestigkeit unzureichend, und der Wandteil vor den Zugrissen gleitet längs einer gekrümmten Fläche ab. Es ist dies nach der Terminologie der Bodenmechanik eine Rutschung oder ein Böschungsbruch.

Wenn die Auflast der Geländestufe die Tragfähigkeit des Vorgeländes am Wandfuß erreicht, wird dieses samt dem Wandfuß in die auf gekrümmter Fläche stattfindende Rutschung mit einbezogen. Eine solche Rutschung wird Grundbruch genannt.

Für unsere Untersuchung von Wänden ist festzuhalten, daß durch die Rutschungen wohl die ursprüngliche Wand zerstört wird, zurückgesetzt sich aber eine neue lotrechte Wand bildet, die nun allerdings höchstens noch die halbe Höhe hat. Die abgerutschte Masse ist dieser Wand bauchig gewölbt vorgelagert. Ganz besonders zu beachten ist aber, daß auch in einer schiefen Böschung durch Rutschungen kleine senkrechte Wände entstehen, da auch hier die Zugrisse vertikal verlaufen.

\section{Wände in Festgestein}

Festgesteine zerfallen weder beim Austrocknen noch beim Durchfeuchten und lassen sich nicht aufschlemmen. Sie zeichnen sich von den bindigen Lockergesteinen durch die um vieles größere Festigkeit aus, so daß Wände von einigen hundert Metern Höhe möglich sind. Es muß aber betont werden, daß von den Lockergesteinen bis zu den Festgesteinen alle Übergänge vorhanden sind, in der einen Richtung z. B. von Sand über alle Verfestigungsstufen bis zum Sandstein, vom Kies bis zum Konglomerat, vom 'Ton bis zu metamorphen Schiefern, umgekehrt aber auch vom kaum zerklüfteten Massengestein bis zum tektonischen Lockergestein oder völlig verwitterten und in Grus zerfallenen Gestein.

Für die Größenordnung, in der Felswände im allgemeinen auftreten, muß ein dem Gestein übergeordneter Begriff, der geologische Körper, verwendet werden. Was am Handstück beobachtet und gemessen werden kann, genügt nicht, um das Verhalten einer Felswand zu verstehen. So gelten z. B. die an einem Gesteinsprobekörper festgestellten Werte der Druck- und Zugfestigkeit nur bedingt für den geologischen Körper, der oft aus verschiedenen Gesteinen zusammengesetzt ist und von Schichtflächen und Schieferungen struiert und von Kluftflächen durchsetzt ist. Durch die Schicht- und Schieferungsflächen wird das Verhalten des geologischen Körpers anisotrop, die Klüfte unterteilen ihn in Einzelkörper. Müller (1963) schlägt für den Zerlegungsgrad des geologischen Körpers die Begriffe «Einkörpersystem», «Mehrkörpersystem», «Vielkörpersystem»und «gekörnte Masse»(tektonisches Lockergestein) vor.

\section{Strukturflächen und Strukturlinien}

Gewisse Eigentümlichkeiten einer Felswand sind rom Verlauf der Schicht-, Schieferungsund Kluftflächen abhängig. Sie sollen, wenn sie sich in der Formung der Oberfläche auswirken, in ihrer Gesamtheit Strukturflächen genannt werden. In einer Wand können sich verschieden- 
artige und verschiedengerichtete Strukturflächen geltend machen. Im folgenden soll ein System, das zudem als durchgängig ebenflächig angenommen wird, in einer generellen Übersicht dargestellt werden. Die Übersicht bezieht sich zunächst ganz allgemein auf ein Talgehänge. Begriffe wie «Längstalgchängc»lassen sich sinngemäß auch als «Längstalwand» verwenden.

Für die Formung des Gehänges ist die Art des Ausstreichens der Strukturflächen, der Schnitt mit der Gehängefläche, von Bedeutung. Die Schnittlinie wird Strukturlinie genannt.

Sind die Strukturflächen horizontal, so verlaufen die Strukturlinien im Gehänge, wie auch dieses gerichtet sei, immer horizontal. Die Struktur des Gehänges ist richtungsunabhängig, das Gehänge hat Horizontalstruktur. Das klassische Beispiel ist der Grand Canyon des Colorado.

Sind die Strukturflächen geneigt, so kann von ihnen eine Streichrichtung angegeben werden. Verläuft ein Tal in der Streichrichtung, so ist es ein Längstal. Beim Quertal verläuft das Tal senkrecht dazu, beim Diagonaltal ist der Winkel der Talachse zur Streichrichtung größer als Null und kleiner als $90^{\circ}$.

Sinngemäß kann von Längstal-, Quertal- und Diagonaltalgehänge und -wänden gesprochen werden. Hier ist nun aber zu beachten, daß völlig parallele Talhänge geometrisch nur möglich sind, wenn das Tal kein Gefälle hat. Mit zunehmendem Gefälle streben auch die Gehänge talauswärts immer stärker auseinander. Das heißt, daß Talhänge nie reine Längstalhänge sein können, wenn die Talachse genau im Streichen liegt.

Bei einem reinen Längstalhang verlaufen die Strukturlinien immer horizontal. Je nach dem Einfallen der Strukturflächen in bezug auf das Gehänge können unterschieden werden:

1. Der Strukturflächcnhang, wenn die Strukturflächen im gleichen Sinn wie der Hang geneigt sind.

a. Die Strukturflächen werden unterschnitten, wenn der Hang steiler ist als die Strukturfläche.

b. Beim Plattcnschußgchänge bilden Strukturflächen die Hangoberfläche.

c. Ist der Hang weniger steil als die Strukturflächen, so werden die Strukturflächen überschnitten.

2. Beim Hang mit V'ctikalstruktur verlaufen die Strukturflächen vertikal.

3. Beim Strukturkopfhang sind Hang und Strukturflächen gegensinnig geneigt. Der Winkel zwischen beiden kann spitz, ein Rechter oder stumpf sein.

Beim Diagonal- und Quertalgchänge sind die Strukturlinien im Gehänge geneigt, sie steigen talabwärts im Gehänge auf oder ab. Es können Strukturkopfdiagonalgehänge und Strukturflächendiagonalgchänge unterschieden werden. Das reine Quertalgehänge bildet den Übergang zwischen beiden.

Da kein Gehänge völlig ebenflächig ist, vielmehr Krümmungen, Einmuldungen (im morphologischen Sinn), Ausbuchtungen und Einschnitte darin vorkommen und die Streichrichtung der Gesteine in einem ausgedehnten Gehänge nicht konstant ist und endlich auch Verfaltungen, Überschiebungen und Verwerfungen auftreten können, so wechseln in der gleichen Gehängeflucht reine Längstalgehängepartien oftmals mit Diagonaltal- und Quertalpartien.

Die Beobachtung zeigt, daß in Hängen mit Horizontal- und Vertikalstruktur, aber auch in Strukturkopfhängen von der Struktur im großen kaum beeinflußte ebenflächige und vertikale Wände auftreten können. Die Strukturen machen sich dann oft nur in Detailformen bemerkbar. Dies gilt auch für Strukturflächenhänge mit wenig geneigten Strukturflächen. Fallen aber die Strukturflächen steil ein, so entwickeln sich meist zwar sehr steile, aber nicht vertikale Wände, die sehr ebenflächig sind: die Plattenschußwände; oft wird sogar besser von Plattenschußfelshang gesprochen.

\section{Verwitterung und Rückwitterung von Felswänden}

Bei der Rückwitterung können vorbereitende Vorgänge und das eigentliche Rückwittern durch abstürzendes Material unterschieden werden. $\mathrm{Zu}$ den vorbereitenden Vorgängen gehört die Gesteinsverwitterung im engern Sinn, bei dem das Festgestein gelockert und oft chemisch verändert wird.

Die Verwitterung von Steilhängen unterscheidet sich grundsätzlich von derjenigen der Flachhänge, da sich kein tiefgründiges Verwitterungsmaterial anhäufen kann. Es kann sich kein Boden bilden. Regenwasser läuft rasch ab, so daß die Verwitterung auch im humiden Klima gewisse aride Züge aufweist.

In den Breiten der Alpen spielt auch die Exposition eine große Rolle, besonders in höhern Lagen, wo Nordwände viel länger vereist sind als besonnte Wände und in Steilhängen bis zu über $50^{\circ}$ Schnee haften bleibt. Schneebedeckte Steilhänge sollen aber in dieser Arbeit nicht berücksichtigt werden.

Durch die Oberflächenverwitterung wird die oberste Schicht von wenigen Millimetern bis zu einigen Zentimetern in der Farbe sichtbar verändert, ein Zeichen der biolo- 
gischen urd chemischen Vorgänge. Beim Anschlagen mit dem Hammer tönt die Oberfläche oft hohl. Manchmal ist der Vorgang in die Tiefe fortgeschritten, und unter einer obersten Platte hat sich eine zweite, ja dritte gelockert. Auch wenn die einzelnen Platten und Schuppen nur locker verbunden scheinen, sind sie oft mit den benachbarten verzahnt. Manchmal genügt aber ein Hammerschlag, um ein ganzes Nest zum Zusammenbruch und Absturz zu bringen. Die äußersten Schuppen, Platten oder Schalen sind völlig ausgetrocknet (ohne Bergfeuchtigkeit) und klirren beim Anschlagen. Sind sie der direkten Sonnenbestrahlung ausgesetzt, so erwärmen sie sich erstaunlich hoch, da sie durch den Luftzwischenraum isoliert sind und die Wärmeableitung ins Gesteinsinnere gering ist.

Mit der Höhe nimmt an günstig exponierten Wänden der Strahlungseinfluß zu. I. Dirmhirn (1952) hat an einer Gneisplatte auf $3050 \mathrm{~m}$ Höhe mit ca. ${ }^{1 / 4} \mathrm{~m}^{2}$ Fläche und ca. $6 \mathrm{~cm}$ Dicke, die dem gewachsenen Fels mit 2-3 cm Luftzwischenraum locker auflag, bei schwachem $W$ ind im September eine Maximaltemperatur von $29^{\circ} \mathrm{C}\left(6,3^{\circ}\right.$ in der Hütte) und eine Minimaltemperatur von $-4,0^{\circ}$ ( $1,8^{\circ}$ in der Hütte) gemessen. Solche vom gewachsenen Fels abstehende Platten spielen bei der Wandverwitterung eine große Rolle. I. Dirmhirn meint: «Es ist also anzunehmen, daß der Zerfall des Gesteins in feinsten Gneisstaub um so rascher vor sich geht, je dünner die Plättchen sind.»

Der Einfluß der Insolation für sich allein wurde aber, wie verschiedene Versuche unter extremen Bedingungen mit fast 90000 Wechseln von $32^{\circ}$ auf $1+2^{\circ}$ zeigten, stark überschätzt. Mit den Wärmeschwankungen sind aber auch Veränderungen der chemischen Vorgänge, z. B. der Hydratation von Salzen (Wilhelmy 1958), die sich beim Verwittern bilden, verknüpft. Hydratation ist mit Volumvergrößerung und damit mit Sprengwirkung verbunden.

Zum thermischen Zerfall gehört indirekt auch die Frostsprengung, welche in alpinen Verhältnissen von ausschlaggebender Bedeutung ist. Die Kleinklüfte und Haarrisse können Wasser speichern, das beim Gefrieren sprengend wirkt. Für poröses Lockermaterial wurden schon die Ansichten von Föppl (1958) wiedergegeben, die hier noch einmal in Erinnerung gerufen werden sollen.

Die Beobachtung zeigt, daß ständige Durchnässung einer Wand, z. B. unter einem Wasserfall, zu keiner erhöhten Verwitterung führt.

Die Verwitterung in einer Wand weist typische Grenzerscheinungen auf. Am Fuß der Wand wird die Verwitterung durch die anliegende Schutthalde beeinfluf.t. Aus dieser kann Feuchtigkeit in den Wandfuß aufsteigen, welche die Verwitterung beschleunigt. Am Wandfuß entstehen durch diese raschere Rückwitterung oft kleine Überhänge. Am Scheitel der Wand kann die Verwitterung überall dort, wo ein Flachhang den obern Abschluß bildet, die tiefgründige Oberflächenverwitterung in das Anstehende übergreifen. Die obersten Meter einer Wand unterliegen deshalb einer besonderen Verwitterung. Dies unterscheidet gratlose Felswände grundsätzlich von gratbildenden Felswänden.

\section{Die Talklïftung}

Von Tunnel- und Stollenbauten ist der Bergschlag bekannt. Wird in einem hochüberlagerten massiven Gestein ein Hohlraum geschaffen, so entstehen um diesen ganz neue Spannungsverhältnisse. Sie können sich als Bergschläge auswirken, wobei schußartig von den Wänden schalige Platten abgesprengt werden. Diese verbiegen sich dabei oft, ohne zu brechen, so daß sie nicht mehr in die Ablösungsstelle passen. An ähnliche Verhältnisse muß bei der Talbildung gedacht werden, da auch hier durch den Abtrag das Gestein entlastet wird und sich die Spannungsverhältnisse an der Oberfläche verändern. Auch beim Wegschmelzen der großen Eismassen der Glazialzeiten veränderten sich die Druckverhältnisse im Talgrund. Endlich ist auch an die Veränderung der thermischen Verhältnisse zu denken, wenn Gestein aus dem Gebirgsinnern an die Oberfläche gelangt. Diese Restspannungen können unter Bildung mehr oder weniger ober- 
flächenparalle!er Klüfte ausgelöst werden. Von Kieslinger (1958) wurde die Erscheinung eingehend beschrieben. Die Klüfte schneiden Schichtungs- oder Schieferungsflächen und passen sich diesen nur an, wenn die Oberfläche mehr oder weniger parallel dazu verläuft. Die Talklüftung reicht, wie Stollenbauten zeigen, 30-40 m tief ins Gebirgsinnere. Die Klüftung macht jede Neigungsveränderung der Oberfläche mit und verläuft z. B. auf Terrassen horizontal. Kieslinger beschreibt einen Fall, bei dem von horizontaler Oberfläche eine Platte in die Höhe geschleudert wurde, wie wenn eine Sprengung stattgefunden hätte.

Als beim Bau der Zentrale Handegg an der Grimsel ein Gneisrundhöcker angeschnitten wurde, lösten sich plötzlich explosionsartig entlang einer senkrechten Fläche ca. $100 \mathrm{~m}^{3}$ Gestein (Cadisch 194t). Die Trümmer waren vorwiegend plattig.

Durch die Talklüftung wird das parallele Rückwittern der Felswände gefördert. Da die Entlastung am Fuß hoher Felswände am größten ist, muß auch die Talklüftung hier am wirksamsten sein. L. Müller (1961) hat ein Bild der Spannungslinien in einer Taleinkerbung gezeichnet, in welchem die größte Dichte über dem Sockel der Wand auftritt. Das Lossprengen der Platten schreitet danach von unten nach oben fort. Beim Rückwittern wird die Steilheit der Wand immer wieder regeneriert.

\section{Neubildung der oberflächenparallelen Klüften}

Die Talklüftung ist nicht ein einmaliger Vorgang, der mit der Tiefenerosion abgeschlossen ist. Vielmehr zeigen Modellversuche zur Klärung der Spannungsverhältnisse in Talhängen (Scheiblauer 1963), «daß erwartungsgemäß im untern Drittel der untersuchten Böschungen die Schubspannungen am größten sind. Gleichzeitig stellte sich heraus, daß in diesem Bereich eine der beiden Hauptnormalspannungen Zugspannungen aufweist.» Daraus wird gefolgert: «Durch Überwindung der an sich relativ geringen Zugfestigkeit eines geklüfteten Gebirgskörpers knickt die Böschung im untern Drittel nach außen aus, und das darüberliegende Gestein bricht dadurch nach.»

Die Beobachtung an ganz verschiedenartigen Steilwänden zeigt immer wieder das gleiche Bild: Die Verwitterung im weitesten Sinn beginnt am Wandfuß und schreitet von hier nach oben fort. Der rasche Fortschritt dieses Vorganges in Kiesgruben und in Lehmwänden wurde weiter oben beschrieben. In viel größern Zeiträumen gilt dieser Vorgang aber auch für Felswände. Die Kluftbildung beginnt am Hangfuß und führt zur Plattenbildung. Diese hängen und reißen nach langer Vorbereitung ab. Manchmal bleiben einzelne Pfeiler stehen, die dachförmige Überhänge abstützen. Die Ausbrüche zeigen oft gewölbeartige Form. Diese ist statisch besonders günstig und bleibt deshalb länger erhalten.

Nach oben nehmen die Spannungen ab. Die Plattenbildung erfaßt deshalb die obersten Partien der Wand nicht. Hier herrschen auch, wie schon erwähnt wurde, andere Verwitterungserscheinungen vor. In den obersten Metern der Wand bleibt der Schutt liegen, ohne abzustürzen. Bei horizontaler Schichtung wird diese herauspräpariert. In vielen Wänden bleiben zuoberst Überkragungen stehen. Man kann diese Erscheinung im Bündnerschiefer der Via Mala ebenso schön beobachtet wie in den Kalkwänden des Lauterbrunnentales (Fig. 1).

Beim Auslösen der Spannungen werden z. T. völlig neue Klüfte erzeugt. Es ist oft überraschend, wie unbekümmert die Plattenabrisse Schichtungs- und Schieferungsflächen schneiden. Wo aber in einem Fels schon steil verlaufende Kluftsysteme vorhanden sind, werden diese aktiviert. Dabei zeigt sich, daß nicht nur Klüfte zur Ausbildung kommen, die parallel der Wandflucht angelegt sind, sondern auch Scherflächen, die unter $45^{\circ}$ zu einer tektonischen Schubrichtung verlaufen. So entstehen in der Felswand Hohlformen, deren beidseitige Flächen sich unter ungefähr $90^{\circ}$ verschneiden und oft nach oben durch ein Dach abgeschlossen sind, aber auch vorstehende Pfeiler.

Damit es in einem Felstabsturz zu diesen verschiedenartigen Erscheinungen kommt, muß die Wand eine bestimmte Höhe erreichen. Es kann ein Unterschied gemacht wer- 


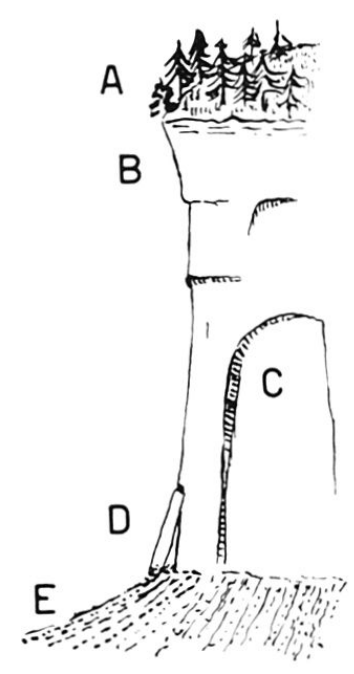

Schema einer Wand

A Deckschicht mit Oberflächenverwitterung, gegen die Felsoberkante etwas abgerundet. B Überkragung, zuoberst mit Verwitterung von der Deckschicht aus. C Nischenausbruch, gewölbeartig abgestützt mit Restpfeiler. D Losgesprengte Platte am Wandfuß. E Schutthalde.

den zwischen kleinen Felsabsätzen, die vorwiegend horizontal struiert sind und bei denen die Oberflächenverwitterung von der Deckfläche her dominiert und wo die senkrechte Plattenbildung keine Rolle spielt, und großen Wänden, wie wir sie eben geschildert haben.

\section{Bildung vertikaler Klüfte. Die «Bergzerreißung»}

Als Festgestein soll auch Eis in die Betrachtung mit einbezogen werden. Die Verwitterung besteht im Abschmelzen. Eine besondere Eigenschaft des Eises ist die Fähigkeit, daß durch Druck Spalten sich nicht nur wieder schließen, sondern wieder zusammenfrieren (Regelation).

Wird ein Gletscher über einen konvexen Gefällsbruch geschoben, so reißt das Eis in meist auffallend regelmäßige Platten mit senkrechter Begrenzung. Nimmt die Bodenneigung stark zu, so überkippen die Platten und zerfallen in Trümmer. Bei Gletscherabbrüchen zu einem natürlichen oder künstlichen Stausee (z. B. Märjelensee oder Grimselsee) bilden sich senkrechte Wände. Die Höhe von Eiswänden in den Polargebieten kann 30 bis $40 \mathrm{~m}$ betragen und in Ausnahmefällen sogar $50 \mathrm{~m}$ übersteigen. Beim Zerfall werden aber nie abgeschrägte Böschungen gebildet. Vielmehr brechen freistehende Platten, Pfeiler oder Türme in sich zusammen und bilden ein grobblockiges Haufwerk. Oft bilden sich Überkragungen im Scheitelbereich.

Auffallend ähnliche Vorgänge und Formen können bei vielen andern Festgesteinen beobachtet werden. Ampferer hat dafür den Ausdruck «Bergzerreißung» geprägt und eine Reihe von Formen beschrieben. Er schreibt 1940: «Die Hauptform der Zerreißung von horizontalen Schichtplatten entsteht durch das Ausweichen weicher Schichtlagen unter dem Drucke der Überlagerung. Die betreffende weiche oder vielleicht auch nur erweichte Schicht wird im Laufe der Zeit mehr oder minder stark zusammengepreßt und nach der freien Seite hinausgeschoben.»

Die durch die Bergzerreißung durch steil verlaufende Spaltflächen abgetrennten Gebirgsteile können wie bei einem Gletscherbruch staffelförmig absacken, oft so allmählich, daß sie nicht zerfallen, unter Umständen aber auch als Bergsturz zu Tal gehen.

Ampferer schreibt 1940 weiter: «Wenn man auf Karten von mindestens 1:25000 arbeitet, so kommt man zu der Einsicht, daß über der ganzen Bergwelt förmlich ein feines Spinnennetz von Zerreißungsfäden ausgespannt liegt ... Die Zerreißungen treten in allen Gesteinsarten auf.»

Es ist zu beachten, daß diese Zerreißungsklüfte sehr steil, meist senkrecht verlaufen und beim Absacken oder Abstürzen der vorgelagerten Felsteile immer wieder neue Felswände bilden, die oft gegen den Sockel zu konkav verlaufen. 
Im Jura mit der häufigen Wechsellagerung verschiedener Gesteinsarten verwittern unter den Kalkfelsen die Mergel- und Tonschichten viel rascher. Durch Wasseraufnahme gehen sie in den plastischen Zustand über und werden dann durch die auflagernden Kalkschichten herausgequetscht. Wenn die Unterlage weicht, werden die überragenden Kalkfelsen auf Zug belastet, es entstehen zunächst Zugspalten, denen entlang dann die Kalkschichten entweder abstürzen oder absacken.

\section{Die Zerstörung von Wänden}

Es wurde gezeigt, daß der Zerfall von Felswänden nicht gleichbedeutend ist mit der Zerstörung der Form, daß sich vielmehr Felswände zerfallend und rückwitternd regenerieren. Einmal muß aber der Zerfall doch zurZerstörung führen. Dabei sind zwei Vorgänge zu unterscheiden, die Erniedrigung von oben und die Aufzehrung der Wand von unten.

Die Wand kann im durchtalten Gebirge nicht unbeschränkt zurückweichen. Auch wenn sich darüber zunächst noch ein Flachhang befindet, nähert sie sich doch einmal dem Gegengehänge, mit dem sie sich verschneidet und zur Gratwand wird, wenn der Gegenhang auch eine Wand ist. Auf den Zerfall von Gratwänden soll hier nicht näher eingetreten werden, nur so viel ist klar, daß Gratwände beim Rückwittern unweigerlich an Höhe verlieren.

Wird am Hangfuß der Wandschutt nicht fortlaufend entfernt, so wächst von unten her eine Schutthalde empor, die zur Gruppe der Flachhänge zu zählen ist. In ihr stürzt das Material nicht mehr ab, es kann nur noch abkriechen, abgleiten und abgespült werden. Meist sind Schutthalden durch einsinkendes Feinmaterial gut verdichtet.

Durch das Höherwachsen der Schutthalde wird immer wieder ein neues Stück der Felswand verdeckt, das dann nicht mehr zurückwittern kann. Unter der Schutthalde bleibt also ein vorstehender Felskern erhalten. Erreicht die Schutthalde den Scheitel der Wand, so ist der Vorgang abgeschlossen.

Die Form des Felskerns unter der Schutthalde wurde von Fisher (1866), Lehmann (1933) und Bakker und Le Heux (1946) theoretisch untersucht. Um das Problem rechnerisch zu bewältigen, müssen bestimmte Annahmen gemacht werden. Fisher und Lehmann gehen davon aus, daß die noch freie, unbedeckte Wand über der Schutthalde parallel zurückwittere. Fisher nimmt weiter vereinfachend an, daß das Schuttvolumen dem abstürzenden Felsvolumen entspreche. In diesem Fall ergibt die Rechnung für den Felskern ein parabelförmiges Querprofil. Erreicht die Schutthalde den Felsscheitel, so bildet ihre Oberfläche eine Tangente an die Parabel. Lehmann verallgemeinerte die Theorie, indem er das Volumen des Schuttes demjenigen des sich lösenden Felsens nicht mehr gleichsetzte. Weicht das Volumen des Schuttes von demjenigen des Felsens ab, so ergibt sich für das Profil des Felskerns eine parabelähnliche logarithmische Kurve, zu der auch hier die Schutthaldenoberfläche im Endzustand die Tangente bildet. Wird am Fuß der Schutthalde immer aller Schutt entfernt, der nachgleitet, ohne daß der Felskern selbst zurückerodiert wird, so entsteht unter einer dünnen Streu von Wandschutt eine ebene Felsfläche mit der Maximalneigung der Schutthalde. Es ist dies nach der Terminologie von Lehmann die Richtersche Denudationsböschung.

Bakker und Le Heux (19+7) versuchten die Theorie von Fisher und Lehmann nochmals zu erweitern, indem sie von der Annahme ausgingen, beim Rückwittern verflache sich der Steilhang zunehmend. Sie stützten sich dabei auf eine Skizze von Philippson (1931) aus dessen «Grundzüge der allgemeinen Geographie». Sie nannten ihre Annahme «rektilineare Rückwitterung von Gehängen». Nach dem, was weiter oben mitgeteilt wurde, widerspricht aber diese Annahme meinen Beobachtungen. Die Eigentümlichkeit der freien Wandverwitterung besteht ja gerade darin, daß die Felswand nicht abgeschrägt und nicht langsam flacher wird, sondern steil, wenn nicht senkrecht bleibt. Die Theorie der rektilinearen Rückwitterung behandelt einen Sonderfall, zu dem mir 
keine Beobachtungen bekannt sind. Lehmanns Theorie der parallelen Rückwitterung vermag die tatsächlichen Verhältnisse besser wiederzugeben.

Die Lehmannsche Felskurve ist als Idealfall zu betrachten. Sie beruht auf der Annahme, daß die Rückwitterung ein stetiger Vorgang sei und die Schuttlieferung in klein. sten Portionen vor sich gehe. Diesem Schema vermöchte die Rückwitterung einer Kiesgrubenwand an heißen Tagen noch am besten zu entsprechen, wenn fast ununterbrochen feiner Schutt von den Wänden rieselt. Nur ist hier die Bestimmung eines «Felskernes» wenig sinnvoll. Die Felswände entledigen sich ihrer Lockermassen oft in katastrophalem Ausmaß, wenn ganze Wandpartien und große Überhänge zusammenbrechen. Sobald aber Schutt in so großer, bergsturzartiger Menge niederfährt, fließt das Material, und die Ablagerungen sind nicht mehr ebenflächig, oft weisen sie auch eine erstaunlich wenig geneigte Oberfläche auf.

Clar (1963) zeigt auch, daß in einem stark zerrütteten Felskörper der Zerfall nicht nur durch Abstürzen, sondern durch «eine Unmenge kleiner Verstellungen» vor sich gehen kann, so daß durch Kriechen und Hakenwerfen im großen von einer plastischen Verformung gesprochen werden kann. L. Müller (1963) spricht bei solchen Vorgängen von Bruchfließen und zeigt, daß dann Gleitvorgänge wie in plastischem Ton auftreten. Solche Vorgänge lassen sich mit der Lehmannschen Theorie natürlich nicht mehr erfassen.

\section{Die innere Gliederung der Wände}

Es können Gliederungen verschiedener Größenordnungen unterschieden werden. Von den Kleinformen der Schuppen (Schalen, die bei der Verwitterung entstehen und Dezimetergröße erreichen können) und den großen Platten, die sich von der Wand lösen und einige Meter groß sind, war schon die Rede.

Hier sollen Formen besprochen werden, welche ganze Wände oder doch große Teile davon meist auffallend geradlinig durchziehen. Sie können horizontal, diagonal oder vertikal angeordnet sein.

Bei den horizontal und weitgehend auch bei den diagonal angeordneten Formen handelt es sich zur Hauptsache um die Herausarbeitung von Unterschieden in der Verwitterungsresistenz. Es wechseln, mehr oder weniger treppenartig übereinander angeordnet, Felswände, oft nur Felsabsätze geringer Höhe, mit bandartig angeordneten Flachhängen, je nach der Geschwindigkeit der Rückwitterung und nach der Höhenlage mit losem Gehängeschutt überzogen oder bewachsen und sogar bewaldet, in großer Höhe mit Schnee oder Eis bedeckt. Die Durchschnittsneigung des Gehänges kann dann alle möglichen Werte annehmen, im Einzelnen besteht aber der Hang aus sehr steilen bis senkrechten Felsabstürzen und Flachhängen mit Neigungen, die den maximalen Winkel der Schutthalden nie überschreiten, d.h. die zwei Flächenarten sind durch scharfe Knicke voneinander abgesetzt.

Um einen ganz andern Formkreis handelt es sich bei den vertikalen Gliederungen. Einmal gehören hierhin die Klüfte, welche durch senkrecht verlaufende Bergzerreissung entstanden sind und die weiter oben schon besprochen wurden, dann all die Brüche, welche mit der Gebirgsbildung zusammenhängen und quer zum Streichen verlaufen. Brüche halten oft gleichmäßige Abstände inne, so daß die Gliederung der Wand auffallend regelmäßig erscheint.

Diese Klüfte sind in ihrer ersten Anlage nur schmal. Durch die Verwitterung aber werden sie zu Kluftgassen erweitert (besonders natürlich, wenn eine enge Scharung von Klüften das Gestein zerrüttet hat). Breite Steinschlagrinnen entstehen, die auch durch das abfließende Wasser trichterförmig erweitert werden, so daß dazwischen nach oben spitzzulaufende Dreiecksfelsformen übrigbleiben. Sind die Abstände größer, so erscheint die Felswand bastionsartig gegliedert. Die Bastionen treten oft abgerundet vor.

Der Einfluß der Abspülung durch Regen darf nicht unterschätzt werden. Zwar sind nach den mir bekannten Abbildungen auch Wände in aridem Gebiet fein ziseliert 
durch oft engständige, senkrecht verlaufende Verwitterungsrinnen, aber diese sind weit weniger trichterförmig erweitert als im humiden Klima. Auch einzelne Pfeiler bleiben in streng senkrechter Form erhalten, während sie in humidem Klima nach oben oft charakteristisch konisch aus!aufen. Dies gilt auch für die «Erdpyramiden», die ja eben keine kantig zugeschnittenen Pyramiden sind, sondern sehr s:eile Kegel (wenn sie nicht durch einen Gipfelblock geschützt sind).

Im humiden Klima sind Felsgipfelformen nicht nur als Ganzes dreieckförmig, sondern durch eine Vielfalt von dreieckförmigen Vorbauten, durch Erosionstrichter getrennt, gegliedert. Bis zum Extrem geht diese Dreieckgliederung in leicht verwitterbarem Material vor sich, wie z. B. beim Pizzo Corombe in der Nähe des Lukmaniers im zuckerkörnigen Dolomit.

\section{Rückblick}

Es gibt Felswände, bei denen es kaum Schwierigkeiten bereitet, die Entstehung zu verstehen. Da sind einmal die Schluchtwände, welche durch das fließende Wasser herausgeschnitten wurden und bei denen noch bis hoch hinauf die Spuren der Arbeit des Wassers zu sehen sind. An andern Orten ist die Unterschneidung des Hanges durch einen Fluß offensichtlich, so daß die Wand durch Nachstürzen entstanden ist. Auch durch den Gletscher werden Wände gebildet, an denen oft noch sehr schön die Gletscherschliffe zu sehen sind. Andere Wände sind so ebenflächig, daß es sich offensichtlich um alte tektonische Bruchflächen handelt, die freigelegt wurden. Oft können noch die Rutschharnische gesehen werden. Viele Felswände sind nicht mehr an der Stelle, wo sie zuerst angelegt wurden. Sie sind als Felswand zurückgewittert. An dieser Rückwitterung sind offensichtlich wie bei den Wänden in kohärentem Lockermaterial zwei Gruppen von Vorgängen beteiligt, die sich durch ihre Größenordnung unterscheiden, wenn sie wohl auch durch Übergänge verbunden sind. Zur Gruppe der Kleinvorgänge ist zunächst und vor allem die Wandverwitterung im engern Sinn, die chemische Zermürbung des Gesteins, die Hydratation, die Frostsprengung und Insolation, die biologische Verwitterung durch Bakterien, Strahlenpilze, Flechien, Moose und Wurzelsprengung durch höhere Pflanzen (Blöchliger 1933) zu zählen. Sie führt zur Abschuppung, Abblätterung bis zur Lossprengung von Platten. Hierher gehören weiter zum größten Teil die Rückwitterung durch Auslösung von Restspannungen und von sich immer wieder neu auslösenden Spannungen am Fuß großer Wände, durch welche flächenparallele Platten losgesprengt werden und abstürzen. Diese Kleinvorgänge, welche größenmäßig das Absanden, den Steinschlag, aber auch den Absturz großer Platten und plattiggelöster Wandpartien umfaßt und oft charakteristisch vom Felswandfuß nach oben fortschreiten, ergeben in ihrer Gesamtheit eine oberflächenparallele Rückwitterung von der Schutthalde bis hinauf zum Scheitel oder bis zur Überkragung.

Dieser Gruppe stehen die Großvoryänge gegenüber. Hierüber gehören die Bergzerreißung, durch welche Felsmassive von oben bis unten gespalten werden und an sehr steilen Flächen auseinanderklaffen. Bei der Bergzerreißung öffnen sich Zugspalten von oben nach unten. Einzelne Partien zerbrechen und zerfallen in ein großblockiges Haufwerk. Häufig sind aber damit Gleitvorgänge verbunden, die beschleunigt als Bergstürze, viel häufiger als Sackungen, vor sich gehen. Die zuerst sich bildenden Zugspalten verlaufen dann wohl sehr steil bis senkrecht, die Flächen aber, auf denen die Massen aus dem Stand gleiten, sind konkav gebogen. Wird die ganze Fläche freigelegt, so geht die steile Felswand in eine rundlich nach außen gebogenen Fußfläche über, die allerdings immer noch so stark geneigt ist, daß sie zu den Felswandformen gehört. Es wurde gezeigt, daß die Höhe standsicherer vertikaler Wände vom Material abhängt. Jirousek (Müller 1963) hat für bestimmte Annahmen standfeste Formen berechnet, bei denen unter einem stabilen vertikalen Teil die Wand leicht konkav ausbiegt. Offenbar wird, wenn die Standsicherheit der vertikalen Form überschritten wird, durch die Rückwitterung eine Wand mit konkavem Sockel angestrebt. 
Zur Gruppe der Großvorgänge gehören auch alle tektonischen Vorgänge, welche zur Wandbildung führen, auf die hier aber nur hingewiesen sei (Lehmann 1941).

All diese Vorgänge sind singuläre Verwitterungsvorgänge, die sich nur in Felswänden abspielen, diese aber immer wieder regenerieren bis zu ihrem restlosen Zerfall, der in einer Erniedrigung der Wandhöhe besteht.

Wir haben am Anfang die Gehänge in Flach- und Steilhänge eingeteilt und die Wände den Steilhängen zugeordnet und als bedeutungsvollen Grenzwert die Maximalböschung von Schutthalden angegeben. In der großen Formgruppe der Flachhänge können alle möglichen Hangneigungen beobachtet werden, von ca. $30^{\circ}$ abwärts bis zur Horizontalen, und zwar in jeder beliebigen Ausdehnung.

Bei den Steilhängen herrschen sehr steile Wände, etwa von $60^{\circ}$, eher von $70^{\circ}$ an aufwärts, vor. Zwischen der Schutthaldenneigung und der Felswandneigung gibt es eine Formlücke. Eine Ausnahme bilden die Plattenschußfelshänge, bei denen die Schichtung oder Schieferung so steil einfällt, daß auch die freie Felsverwitterung mit immer wieder neuer Entblößung durch rasch abrutschendes Material ihnen parallel verläuft. Hier ist es möglich, daß Plattenschußfelshänge in der Neigung unmerklich in Schutthalden übergehen. In der Natur aber ist der Übergang doch meist scharf markiert, weil in der Vegetationszone die Schutthalden besiedelt werden können und sie sich dann durch ihre andere Farbe von den Plattenschüssen abheben. Bei allen andern Hängen, die scheinbar in die Lücke einzureihen sind, handelt es sich um Durchschnittsneigungswinkel eines mehrfach getreppten Hanges, der aus Wandabstürzen und Flachabsätzen zusammengesetzt ist.

Es sei hier nochmals an die Langsamkeit der Vorgänge in Felsgestein erinnert. Die vielen Siedlungen, welche seit Jahrhunderten am Fuß von Wänden bestehen und nur selten von vereinzelten Felsstürzen bedroht und noch seltener von Bergstürzen vernichtet wurden, zeigen am besten den säkularen Vorgang der Rückwitterung. Als bemerkenswertes Beispiel möge die Abtei von St. Maurice am Fuß einer Wand dienen, die auf das Jahr 515 zurückgeht und mehrmals durch Felsstürze beschädigt, doch nie zur Aufgabe gezwungen wurde.

Ferner sei auf die außerordentliche Vielgestalt des ganzen Phänomens Felswand nach Material, Bildung und einwirkenden Faktoren hingewiesen. Ich bin mir bewußt, daß die wenigen hier vorgebrachten Beobachtungen und Ansichten dem Reichtum der Vorgänge bei weitem nicht gerecht werden. Es wird eine reizvolle Aufgabe der Forschung sein, gut fundierte Einsichten in die Dynamik der Felswände zu gewinnen.

\section{LITERATUR}

Ampferer, O.: Zum weitern Ausbau der Lehre von den Bergzerreißungen. Sb. Akad. Wiss. Wien Kl. I 149/19to. Bakker, J.P. und Le Heux, J.W. N.: Projective-geometric treatment of O. Lehmanns Theory of the transformation of steep mountain slopes. Proc. Koninkl. Nederl. Akad. van Wetenschappen 19+6; - Theory on central rectilinear recession of slopes. Proc. Kon. Nederl. Akad. van Wetenschappen 1947. Bernatzik, $W$.: Baugrund und Physik, Zürich 19+7. Blöchliger, G.: Kleinlebewesen und Gesteinsverwitterung. Zschr. f. Geom. 1933. Cadisch, J.: Die Geologie im Dienste des alpinen Kraftwerkbaues. Techn. Rundschau 194t. Clar, E.: Gefüge und Verhalten von Felskörpern in geologischer Sicht. Felsmechanik und Ingenieurgeologie, Vol. I 1963. Dirmhirn, L: Studie über die Oberflächentemperatur fester Körper. Wetter und Leben 1958. Fisher, O.: On the desintegration of chalk cliff. Geol. Mag. 3 1866. Freise, F.W.: Beobachtungen über Erosion an Urwaldgebirgsflüssen des brasilianischen Staates Rio de Janeiro. Zschr. f. Geom. 1932/33. Gerber, E.: Form und Bildung alpiner Talböden. Geogr. Helv. 1959. Guillien, Y.: Monographie d'une paroi de sablière (1935-1959). Zeitschr. f. Geom. Suppl. Bd. I, 196o. Kieslinger, A.: Restspannung und Entspannung im Gestein. Geol. und Bauwesen 1958. Föppl, L.: Die Sprengwirkung des Wassers beim Druckversuch mit wasserhaltigen Feststoffen. Geol. und Bauwesen 1958. Lehmann, O.: Morphologische Theorie der Verwitterung von Steinschlagwänden. Viertelj. schr. Natf. Ges. Zürich 1933; - Die morphologische Wirksamkeit und topographische Verborgenheit von Verwerfungen. Vierteljschr. d. Natf. Ges. Zürich 1941. Mortensen, H.: Zur Theorie der Formentwicklung freier Felswände. Zschr.f. Geom. Suppl. Bd. 1 1960. Müller, L.: ̈̈ber die Entstehung oberflächenparalleler Klüfte. Versuch einer geomechanischen Erklärung. Geol. u. Bauwesen 1961; 
- Die Standfestigkeit von Felsböschungen als spezifisch geomechanische Aufgabe. Felsmechanik und Ingenieurgeologie Vol. I 1963. Philippson, A.: Grundzüge der allgemeinen Geographie II 1931. Piwowar, A.: Über Maximalböschungen trockener Schuttkegel und Schutthalden. Zürich 19o3. Scheiblauer, J.: Modellversuche zur Klärung des Spannungszustandes in steilen Böschungen, Felsmechanik und Ingenieurgeologie 1963. Scheidegger, A.E.: On the tectonic stresses in the vicinity of a valley and a mountain range. Royal Soc. of Victoria 1963. Stini, J.: Neigungswinkel von Schutthalden. Zschr.f. Geom. 1925/26; - Unsere Täler wachsen zu. Geol. und Bauwesen 1942. Terzaghi, K.: Theoretische Bodenmechanik. Berlin 1954. Wilhelmy, Herbert: Klimamorphologie der Massengesteine. Braunschweig 1958. Jaccklin, F.P.: Die Berechnung von Felswänden und Überhängen. Manuskript 1963.

\section{FORMATION ET DESAGREGATION DE PAROIS}

Les parois se trouvent non seulement dans les roches solides, mais peuvent exister dans chaque roche meuble cohérente. Pour le matériel cimenté il n’y a aucune pente maximale, mais une hauteur critique pour des parois abruptes. Comme les processus se développent plus vite dans le matériel meuble que dans la roche solide, leur observation pour des paraois rocheuses sont riches en enseignements. La formation et la désagrégation des parois de glace seront également comprises dans cette étude. Les éboulis, en tombant, ne désagrèrent jamais les pa rois jusqu'à la formation d'un sol. L'exfoliation et certains caractères arides sont typiques pour ces deux désagrégations. La fissuration apparaît dans les parois d'une certaine hauteur et provoque l'éclatement de plaques parallèles. Ce processus comparable aux éboulements dans les tunnels et les galéries est provoqué par les tensions internes dûes à la décharge des couches d'érosion et de décomposition.

Les états de tension au pied de grandes parois conduisent graduellement à l'effritement de nouvelles plaques. La décomposition commence au pied de la paroi et se développe parallèlement à la surface de celle-ci, de telle façon qu'elle ne s'aplanit guère. Lors de la formation des grandes parois, les ruptures de la montagne par tension (Bergzerreissung d'après Ampferer) jouent un rôle, provoquant des fissures verticales, le long desquelles se développent soit des glissements de roche soit des affaissements étendus. Les processus décrits se rapportent aux parois de roche sans crêtes.

La multiplicité des formes et les processus dans des parois avec crêtes seront traités plus tard.

\section{FLUGBILD DER SCHWEIZER STADT}

Geistige Landesverteidigung kann von den verschiedensten Ansatzpunkten ausgehen. Je länger je mehr drängt sich dabei auch die Ansicht in den Vordergrund, es sei nicht nur eine politische, sondern ebensosehr eine kulturelle Angelegenheit. Heimatliebe und Heimattreue setzen sich aus unzähligen bewußten Einsichten und unbewußten Regungen zusammen. Im Hinblick auf die Schweizerische Landesausstellung 1964 unternimmt es der Geographische Verlag Kümmerly \& Frey, Bern, dem Schweizervolk ein wissenschaftlich untermauertes Besinnungswerk über seine Städte vorzulegen. Das Vorwort dazu hat Bundesrat Bonvin verfaßt; in die wissenschaftlichen Abhandlungen teilen sich die Professoren Dr. H. Boesch, Zürich, Dr. P. Hofer, Bern und Dr. G. Grosjean, Bern.

Das Werk ist eine zutiefst seelisch-geistige Auseinandersetzung mit dem Begriff der Schweizer Stadt. Wir unterscheiden dabei den historischen, den geographischen und den statistischen Stadtbegriff. Wohl die einfachste Definition ist jene des Statistikers, der von einer bestimmten Einwohnerzahl, z. B. von zehntausend, an die Gemeinde zur Stadt erklärt und sie in Klein-, Mittel- und Großstädte unterteilt. Die Schweizer Statistik weist für 196o fünfundsechzig Gemeinwesen mit über zehntausend Einwohnern auf. Doch zeigt sich sofort, daß dieser statistische Stadtbegriff zu eng ist. Er muß unbedingt durch den historischen und geographischen Stadtbegriff erweitert werden. Der historische Stadtbegriff stützt sich in unserem Lande hauptsächlich auf besondere Rechte, die in früheren Zeiten einem Gemeinwesen verliehen worden sind und es dadurch vor den andern abgehoben hat. Dieses Bewußtsein ist heute noch tief in unserem Volk verankert, obwohl seit der Gründung des Bundesstaates alle Vorrechte aufgehoben sind, alle Gemeinden einander rechtlich gleichgestellt sind. Viele dieser einst bevorrechteten Orte dehnten sich im Laufe der Zeit aus - sie wurden auch gemäß moderner Statistik zur Stadt —, andere stagnierten oder verschwanden sogar gänzlich. Nach geographischer Begriffsbildung sind Städte zentrale Orte einer bestimmten Kategorie mit all den Einrichtungen, die ihnen gestatten, die entsprechen-

1 Boesch Hans und Hofer Paul: Flugbild der Schweizer Stadt, Bern 1963. Kümmerly \& Frey, 252 Seiten, 152 Flugaufnahmen, 11 Kupferstiche nach Matthäus Merian d. Ae., 23 Ausschnitte der Landeskarte 1:25 ooo und 1:50 o0o, 57 Kartenskizzen, 81 Seiten Text, Fr. 58.—. 